RevMexAA (Serie de Conferencias), 00, 田(2000)

\title{
NEUTRON DIFFUSION AND NUCLEOSYNTHESIS IN AN INHOMOGENEOUS BIG BANG MODEL
}

\author{
J. F. Lara \\ Center for Relativity, UT, Austin
}

\begin{abstract}
RESUMEN
El resumen será traducido al español por los editores.

This article describes the production of primordial ${ }^{4} \mathrm{He}$ nuclei in an inhomogeneous universe. The baryon distribution is spherically symmetric and consists of a high density inner region and a low density outer region. As the temperature decreases neutrons diffuse to the outer region until they are homogeneously distributed, and protons may be redistributed depending on how fast diffusion occurs. Nucleosynthesis occurs earlier in the inner region and neutrons diffuse back to that region. The rapidity of diffusion determines how much ${ }^{4} \mathrm{He}$ is ultimately produced.
\end{abstract}

\section{ABSTRACT}

This article describes the production of primordial ${ }^{4} \mathrm{He}$ nuclei in an inhomogeneous universe. The baryon distribution is spherically symmetric and consists of a high density inner region and a low density outer region. As the temperature decreases neutrons diffuse to the outer region until they are homogeneously distributed, and protons may be redistributed depending on how fast diffusion occurs. Nucleosynthesis occurs earlier in the inner region and neutrons diffuse back to that region. The rapidity of diffusion determines how much ${ }^{4} \mathrm{He}$ is ultimately produced.

\section{Key Words: NUCLEOSYNTHESIS - COSMOLOGY - INHOMOGENE- ITY - NEUTRINO HEATING}

\section{INTRODUCTION}

In 1999 the author wrote a big bang nucleosynthesis code corresponding to a universe with an inhomogeneous baryon distribution. The code envisions the universe as a lattice of spheres and treats one sphere as characteristic of the rest of the universe. The sphere has an initial radius ( the distance scale $r_{i}$ ) of 25000 $\mathrm{cm}$. at temperature $T=100 \mathrm{GK}$. The sphere consists of a high density inner region and a low density outer region. The boundary radius $r_{b}$ between the regions is set to $r_{i} / 2$ and the density contrast ratio $R_{\rho}=$ 800:1. The baryon-to-photon ratio $\eta$ in this model is $3 \times 10^{-10}\left(\eta_{10}=3\right)$. The sphere is divided into a core and 63 spherical shells.

The code solve for the number density $n(i, s)$ of isotope species $i$ contained in spherical shell $s$. The evolution of $n(i, s)$ obeys the equation (Kainulainen et al. 1998)

$$
\begin{aligned}
\frac{d n(i, s)}{d t}= & \frac{1}{n_{b}(s)} \sum_{j, k, l} N_{i}\left(-\frac{n^{N_{i}}(i, s) n^{N_{j}}(j, s)}{N_{i} ! N_{j} !}[i j]+\frac{n^{N_{k}}(k, s) n^{N_{l}}(l, s)}{N_{k} ! N_{l} !}[k l]\right) \\
& -3 \dot{\alpha} n(i, s)+\frac{1}{r^{2}} \frac{\partial}{\partial r}\left(r^{2} D_{n} \frac{\partial n(i, s)}{\partial r}\right)
\end{aligned}
$$

The first term accounts for nuclear and weak reactions occuring between isotopes within shell $s$. The second term corresponds to the expansion of the universe. And the last term accounts for diffusion of isotope $i$ between shells, though in the model only neutrons diffuse. 


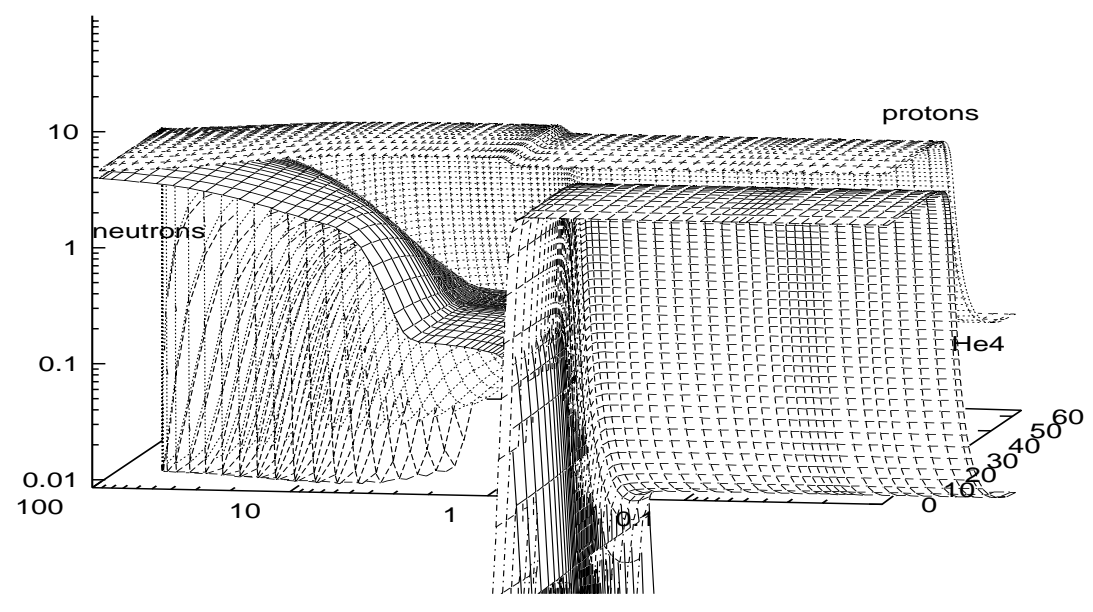

Fig. 1. Number densities of neutrons, protons and $4 \times{ }^{4} \mathrm{He}$
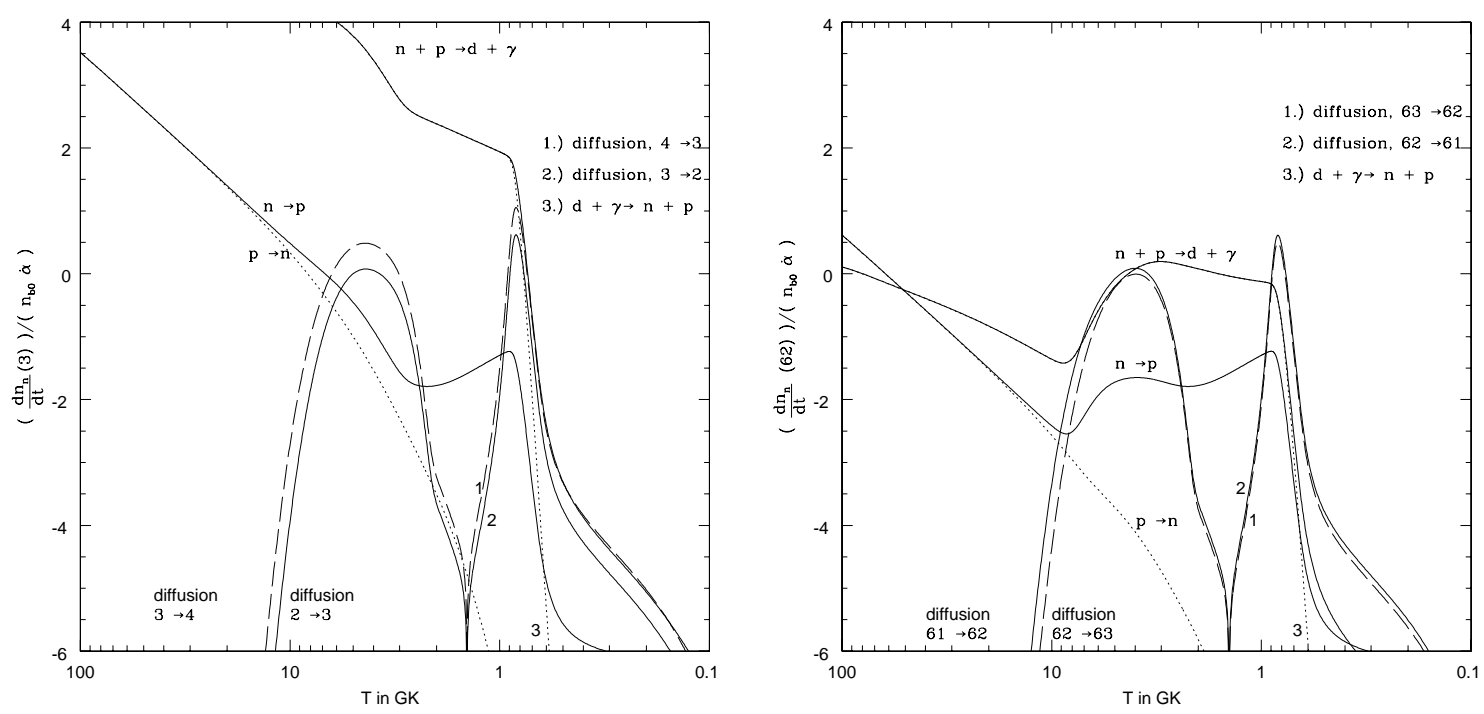

Fig. 2. Neutron Reaction Rates ( shell 3 and shell 62 ) 


\section{RESULTS FOR $R_{I}=25000 \mathrm{CM}, \eta_{10}=3$}

Figure (1) shows the number densities of free neutrons $(n(\mathrm{n}, s))$, protons $(n(\mathrm{p}, s))$ and $4 \times{ }^{4} \mathrm{He}\left(4 n\left({ }^{4} \mathrm{He} e, s\right)\right.$ ). The horizontal axes correspond to the temperature $T$ from 100 GK down to 0.01 GK and the distance scale $r_{i}$ normalized to 64 dimensionless units, and the vertical axis is number density $n(i, s)$ normalized to the initial baryon density $\left(n_{b 0}\right)$. At $T=100 \mathrm{GK}$ number densities in the inner shells vs. the outer shells are large. Weak reactions convert neutrons to protons until they no longer become significant at around $T=13$ GK. Figure (2a) shows the neutron-proton conversion rates for shell 3, a high density inner shell ( normalized to $n_{b 0}$ and to the expansion rate of the universe $\left.\dot{\alpha}\right)$. Figure $(2 \mathrm{a})$ also shows that around $T=6$ GK neutrons start to diffuse significantly into the lower density outer shells. But since the conversion rates are no longer effective at this temperature the protons in the inner shells remain there. At around $T=2.5$ GK the neutrons are homogeneously distributed throughout the whole sphere.

Nucleosynthesis occurs at about $T=0.9 \mathrm{GK}$, when the nuclear reaction $\mathrm{n}+\mathrm{p} \leftrightarrow \mathrm{d}+\gamma$ falls out of equilibrium. Other nuclear reactions then build heavier nuclei, but most baryons wind up as part of ${ }^{4}$ He nuclei. Because of higher density nucleosynthesis occurs earlier in the inner shells, depleting neutrons. So neutrons diffuse back into the inner regions as shown on the right of Figure (2a). Figure (2b) shows the same reaction rates for shell 62, a low density outer shell. The rate for $\mathrm{n}+\mathrm{p} \leftrightarrow \mathrm{d}+\gamma$ is lower and flatter than in Figure (2a). Figure (1) shows that most neutrons back diffuse into the inner shells and then undergo nucleosynthesis, leading to ${ }^{4} \mathrm{He}$ production overwhelmingly concentrated in the inner shells.

The final mass fraction $X^{4} \mathrm{He}$ of the whole model is 0.243664 . This is the result for $r_{i}=25000 \mathrm{~cm}$ and $\eta_{10}=3$ Table (1) shown below lists $X_{{ }^{4} \mathrm{He}}$ for $r_{i}=100 \mathrm{~cm}$ up to $6.3 \times 10^{6} \mathrm{~cm}$. For $r_{i}$ less than $25000 \mathrm{~cm}$ neutron diffusion occurs early enough to coincide at least partly with the time when the conversion reactions are significant. In the inner regions the reactions convert protons into neutrons that then diffuse to the outer regions where they get converted back to protons. But as $r_{i}$ gets larger more protons remain in the inner regions and $X_{4} \mathrm{He}$ increases. $r_{i}=25000 \mathrm{~cm}$ is the turning point when all the protons in the innermost regions remain there. For larger $r_{i}$ the back diffusing neutrons can't reach the innermost regions before nucleosynthesis is completed. So with fewer neutrons in the inner regions $X^{4}{ }_{4 e}$ decreases. At $r_{i}=7.9 \times 10^{5} \mathrm{~cm}$ though diffusion becomes significant at $T=0.9 \mathrm{GK}$, right during nucleosynthesis. For larger $r_{i}$ nucleosynthesis occurs before neutron diffusion and the inner regions, with high proton and neutron densities, produce enough ${ }^{4} \mathrm{He}$ to raise $X^{4} \mathrm{He}$ significantly.

\begin{tabular}{r|c}
$r_{i}$ in $\mathrm{cm}$. & $X^{4} \mathrm{He}$ \\
\hline $10^{2}$ & 0.239029657 \\
$1.58489 \times 10^{3}$ & 0.239369667 \\
$2.51189 \times 10^{4}$ & 0.243663794 \\
$10^{5}$ & 0.241847338 \\
$7.94328 \times 10^{5}$ & 0.229781933 \\
$6.30957 \times 10^{6}$ & 0.250711334
\end{tabular}

Table (1): ${ }^{4} \mathrm{He}$, where $\eta=3.0 \times 10^{-10}$.

\section{CONCLUSIONS AND FUTURE RESEARCH}

Figure (3a) shows $X^{4} \mathrm{He}$ for a range of $\eta$ from $10^{-10}$ to $3 \times 10^{-9}$ as well as the range of $r_{i}$ listed in Table (1) For all values of $\eta$ shown one can see the turning points at which $X^{4} \mathrm{He}$ increases and then decreases and then increases again. The code can generate contour maps for 67 other isotope species as well. Comparing the contour maps of $X_{{ }^{4} \mathrm{He}}, Y(\mathrm{~d}) / Y(\mathrm{p})$ ( $Y(\mathrm{~d})$ is the abundance of deuterium ), and $Y\left({ }^{7} \mathrm{~L} i+{ }^{7} \mathrm{Be}\right) / Y(\mathrm{p})$ to the $2 \sigma$ ranges of recent observations ( IT, 1998; (Izotov \& Thuan 1998) BT, 1998; (Burles \& Tytler 1998) PWSN, 1998 (Pinsonneault et at. 1998)).

$$
X_{{ }^{4} \mathrm{He}}=0.244 \pm 0.002
$$



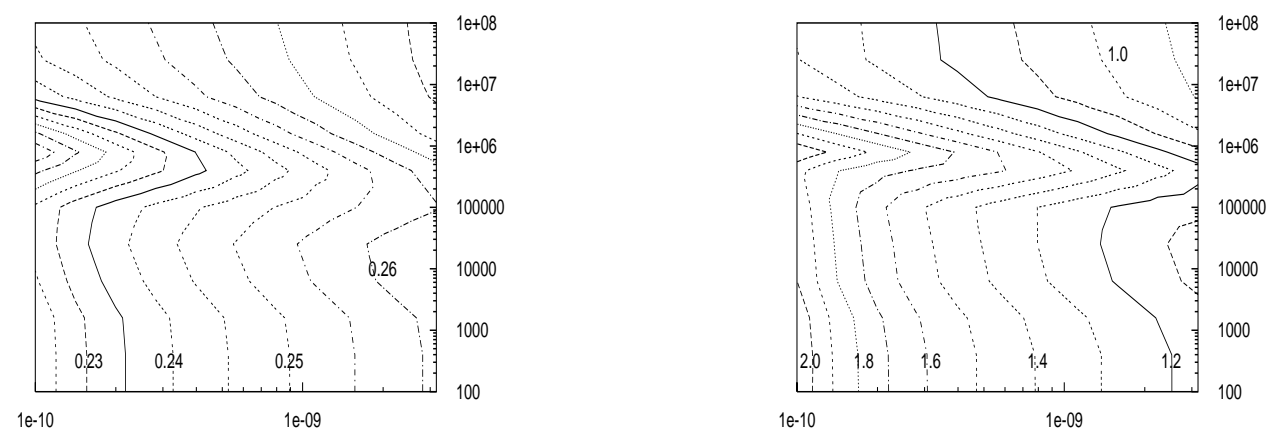

Fig. 3. $X^{4} \mathrm{He}$ and $10^{4} \times \Delta X^{4} \mathrm{He}$

$$
\begin{aligned}
Y(\mathrm{~d}) / Y(\mathrm{~d}) & =(3.4 \pm 0.3) \times 10^{-5} \\
\log \left[Y\left({ }^{7} \mathrm{~L} i\right) / Y(\mathrm{~d})\right] & =-9.45 \pm 0.20
\end{aligned}
$$

the author determined an observational range for $\eta$ from $4.7 \times 10^{-10}$ to $6 \times 10^{-10}$.

A planned article will describe ${ }^{4} \mathrm{He}$ production in greater detail, describing results for values of $r_{i}$ other than $25000 \mathrm{~cm} . R_{\rho}, r_{b}$ and the geometry of the model can be varied in the code. In the future the author will look at results with different values for these parameters. Also, the code has written into it subroutines to calculate the neutrino heating effect. Electrons and positrons annihilate each other from $T=5$ GK to $T=1$ GK. Through annihilation and scattering they can transfer some energy to neutrinos, which in turn can alter the neutron-proton conversion reaction rates and ultimately ${ }^{4} \mathrm{He}$ production. Figure (3b) shows the change $\Delta X_{{ }^{4} \mathrm{He}}$ of the mass fraction of ${ }^{4} \mathrm{He}$ for the same ranges of $\eta$ and $r_{i}$ as in Figure (3a). $\Delta X_{{ }^{4} \mathrm{He}}$ imitates $X^{4} \mathrm{He}$, but not exactly. The change also remains within an order of $10^{-4}$. The author will determine if the change is equivalent to a constant shift of Figure (3a) to the left, and if the coding for the neutrino heating effect is correctly represented in the code.

\section{REFERENCES}

Burles, S. \& Tytler, D. astro-ph/9803071

Izotov, Y. I. \& Thuan, T. X. 1998, ApJ 497, 227

Kainulainen, K., Devine, J. \& Sihvola, E. astro-ph/9807098

Pinsonneault, M. H., Walker, T. P., Steigman, G. \& Narayanan, V. K. astro-ph/9802315

J. F. Lara: University of Texas at Austin, Physics/Relativity, Austin, TX 78712-1081 ( juan@einstein.ph.utexas.edu ). 